\title{
First detection of Leishmania infantum in Phlebotomus kandelakii using molecular methods in north-eastern Islamic Republic of Iran
}

\author{
Y. Rassi, ${ }^{1}$ M.R. Abai', M.A. Oshaghi, ${ }^{1}$ E. Javadian, ${ }^{1}$ A. Sanei, ${ }^{1}$ S. Rafidzadeh, ${ }^{2}$ and K. Arzamani ${ }^{3}$
}

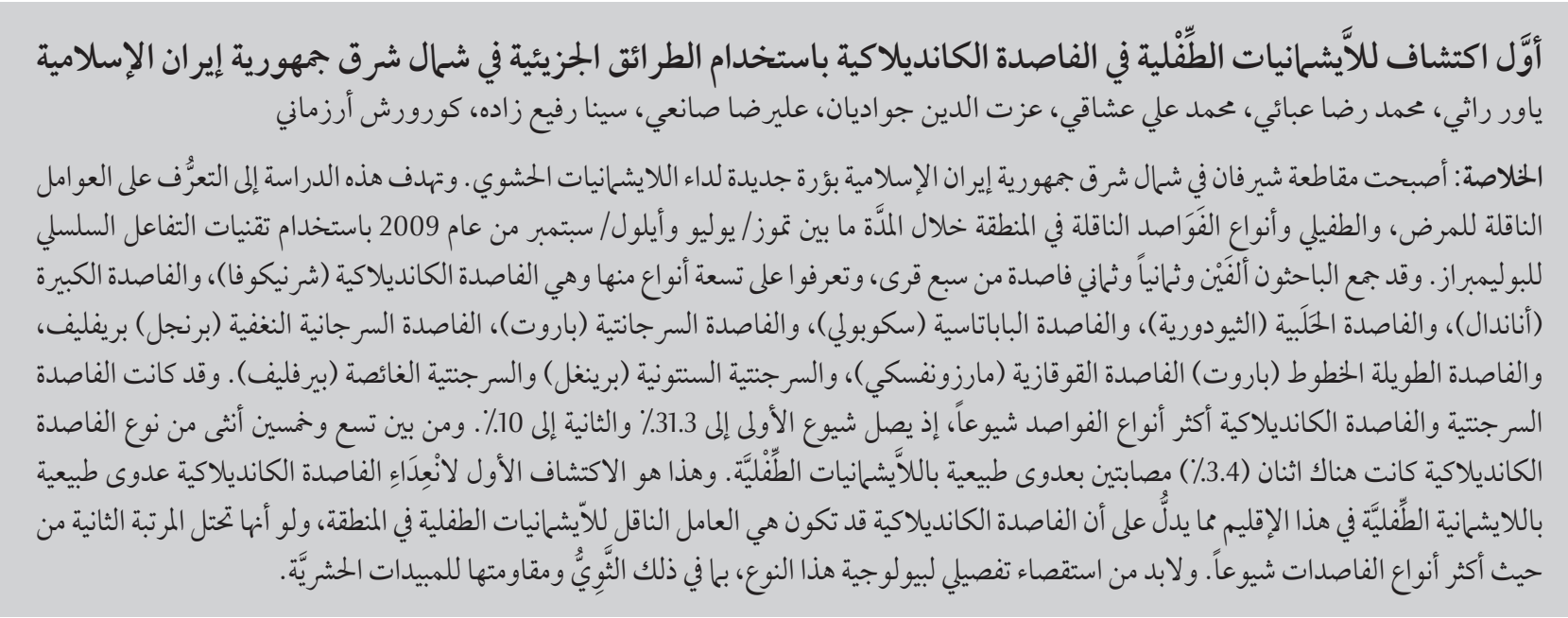

ABSTRACT Shirvan district in north-eastern Islamic Republic of Iran is a new focus of visceral leishmaniasis. This study aimed to identify the vector(s), the parasite and the species composition of sand flies in the district during JulySeptember 2009 using polymerase chain reaction techniques. In all, 2088 sand flies were collected from 3 villages. Nine sand fly species were identified: Phlebotomus kandelakii (Shchurenkova), P. major (Annandale), P. halepensis (Theodor), P. papatasi (Scopoli), P. sergenti (Parrot), P. longidactus (Parrot), P. caucasicus (Marzinovsky), Sergentomyia sintoni (Pringle) and S. sumbarica (Perfil'ev). P. sergenti and P. kandelakii were the most prevalent Phlebotomus species at $31.3 \%$ and $10.0 \%$ respectively. Of 59 female $P$. kandelakii, $2(3.4 \%)$ were naturally infected with $L$. infantum. This is the first finding of natural infection of $P$. kandelakii by $L$. infantum in this region suggesting $P$. kandelakii may be the vector of $L$. infantum in the area although it is the second most prevalent phlebotomine species.

Première détection de Leishmania infantum dans Phlebotomus kandelakii à l'aide de méthodes moléculaires dans le nord-est de la République islamique d'Iran

RÉSUMÉ Le district de Shirvan dans le nord-est de la République islamique d'Iran est un nouveau foyer de leishmaniose viscérale. La présente étude visait à identifier le(s) vecteur(s), le parasite et la composition des espèces des phlébotomes dans le district, de juillet à septembre 2009, à l'aide de techniques d'amplification en chaîne par polymérase. Au total, 2088 phlébotomes ont été prélevés dans trois villages. Neuf espèces de phlébotomes ont été identifiées : Phlebotomus kandelakii (Shchurenkova), P. major (Annandale), P. halepensis (Theodor), P. papatasi (Scopoli), P. sergenti (Parrot), P. longidactus (Parrot), P. caucasicus (Marzinovsky), Sergentomyia sintoni (Pringle) et S. sumbarica (Perfil'ev). P. sergenti et $P$. kandelakii étaient les espèces de phlébotomes les plus répandues (31,3\% et $10 \%$ de l'échantillon respectivement). Sur 59 P. kandelakii femelles, deux (3,4\%) étaient naturellement infectées par $L$. infantum. Il s'agit de la première détection d'une infection naturelle de $P$. kandelakii par $L$. infantum dans la région, suggérant que $P$. kandelakii pourrait être le vecteur de $L$. infantum dans la zone, même si elle n'est que la deuxième espèce la plus répandue de phlébotomes.

'Department of Medical Entomology and Vector Control, School of Public Health, Tehran University of Medical Sciences, Tehran, Islamic Republic of Iran (Correspondence to M.R. Abai: abaimr@tums.ac.ir). ${ }^{3}$ Center of Disease Control, Ministry of Health and Medical Education, Tehran, Islamic Republic of Iran. ${ }^{3}$ North Khorassan University of Medical Sciences, Bojnourd, Islamic Republic of Iran.

Received: 06/10/10; accepted: 02/03/11 


\section{Introduction}

Leishmaniases are a broad spectrum of diseases, ranging from self-limiting localized cutaneous lesions to visceral leishmaniasis (VL) with fatal spontaneous evolution [1]. The visceral form of the disease is the most severe and is nearly always fatal if left untreated [2].

More than $90 \%$ of $\mathrm{VL}$ cases in the world are reported from Bangladesh, Brazil, India and Sudan [3]. VL caused by Leishmania infantum affects approximately half a million new patients each year in the Mediterranean region and Latin America [4]. In the Mediterranean basin, domestic dogs (Canis familiaris) are the principal reservoir host, and some species of sand flies belonging to the subgenus Larroussius are the primary vectors [3].

VLoccurs sporadically throughout the Islamic Republic of Iran. However, there are 4 important endemic foci in the country: Ardebil and East Azerbaijan provinces in the north-west of the country and Fars and Bushehr provinces in the south [5-8]. Two sand fly species, Phlebotomus (Lar.) perfiliewi (Parrot) and P. (Lar.) kandelakii (Shchurenkova) have been shown to be vectors of $L$. infantum in north-western Islamic Republic of Iran [9-11]. The species of P. (Paraphlebotomus) alexandri (Sinton) and $P$. (Lar.) major (Annandale) have been found naturally infected with $L$. infantum and are the VL vectors in the south part of the country [12-14]. P. (Lar.) keshishiani (Shchurenkova) and P. (Para.) caucasicus have been reported infected with promastigotes in southern Islamic Republic of Iran but no parasite identification was carried out $[15,16]$.

This study was carried out to identify the vector(s) and the parasite, as well as the species composition of sand flies in a new VL focus, Shirvan district, in north-eastern Islamic Republic of Iran.

\section{Methods}

\section{Study area}

The study was conducted in Shirvan district, North Khorassan province, northeastern Islamic Republic of Iran. This region is 1350 meters above sea level. The total population of Shirvan district was around 164000 in 2006. The northern part of Shirvan is mountainous with cold weather and the southern part has a temperate climate due to the flow of the Atrak River. The warm season is short (mid-May to mid-September) in the mountainous areas. The main occupations of the population are farming and raising animal.

\section{Sand fly collection}

Based on previous mass screening of dogs using the DOT test and confirmation that canine species as a reservoir of VL in Shirvan district (Mohebali et al., unpublished data, 2008), 3 villages, Gholban, Starkhi and Hossein-abad, were selected. Sand flies were collected biweekly from indoors (e.g. bedroom, guest bedroom, toilet, and stable) as well as outdoors (wall cracks and crevices and animal burrows) by using sticky paper (60 papers per village) during July-September 2009. All traps were installed at sunset and collected near sunrise. The sand fly specimens were washed in $96 \%$ ethanol alcohol to get rid of the sticky materials and to preserve them. Dissection of preserved sand flies was done in phosphate buffered saline (PBS) solution. The terminal segments of the abdomen containing the spermatheca and the heads of females were removed and mounted in a drop of Puri medium and identified to species level using keys of Theodor and Mesghali [17]. The remains of the bodies of the sand flies were kept individually in $96 \%$ alcohol and stored at $-20^{\circ} \mathrm{C}$ for molecular analysis.

\section{DNA extraction}

DNA of the specimens was extracted using the Bioneer Genomic DNA
Extraction Kit (North Korea), according to the manufacturer's instructions. Extraction was carried out on the remaining body of the individual sand fly and stored at $4{ }^{\circ} \mathrm{C}$. Double distilled water and DNA from L. major, L. tropica, and $L$. infantum, provided to the Iranian Institute of Pasteur by the World Health Organization, were used as negative and positive controls.

\section{DNA amplification and PCR-RFLPs}

Primary examination for infection of sand flies with Leishmania species was performed using nested- polymerase chain reaction (PCR) against the minicircle kinetoplast (k)-DNA using the following primers [18]: CSB2XF (forward): 5'-C/GA/GTA/ GCAGAAAC/TCCCGTTCA-3' (20 bp); CSBIXR (reverse): 5' - АТT TT CG/CGA/ TT T/ CGCAGAACG-3' (20 bp); 13Z (forward): 5'-ACTGGGGGTTGGTGTAAAATAG-3' (22 bp); LIR (reverse): 5' -TCGCAGAACGCCCCT-3' (15 bp).

Restriction fragment length polymorphism (RFLP) PCR was done. Positive samples against kDNA were tested against the ribosomal internal transcribed spacer 1 (ITS1) region using the primers LITSR (5'-CTGGATCATTTCCGATG-3') and L5.8S (5'-TGATACCACTTATCGСАCТГ-3') followed by digestion by HaeIII [19].

The PCR products were run along with a 100 bp ladder on $1.2 \%$ agarose gel containing ethidium bromide for $1 \mathrm{~h}$ at $80 \mathrm{~V}$. The gel was observed on a ultraviolet (UV) transilluminator and then digital photographs were prepared. Parasites were identified by comparison with positive controls of $L$. infantum, L. major and L. tropica and molecular weight markers.

We added $2 \mu \mathrm{L}$ HaeII to the ITS 1 PCR products $(20 \mu \mathrm{L})$ at $37^{\circ} \mathrm{C}$ for 12 $\mathrm{h}$ with conditions recommended by the supplier (Fermentas, Germany). The restriction fragments were subjected 
to electrophoresis in 3\% agarose gel containing ethidium bromide $(0.5 \mu \mathrm{g} /$ $\mathrm{mL}$ ) for $3 \mathrm{~h}$ at $65 \mathrm{~V}$ and observed on a UV transilluminator [20,21].

\section{Cathepsin B-like cysteine protease E/F PCR}

Cathepsin B-like cysteine protease (cpb)E/F PCR was done. This PCR is species-specific for $L$. donovani complex and was developed by Hide and Bañuls [21]. cpbE/F amplification was done in $30 \mu \mathrm{L}$ with 6 pmol of each primer (forward: 50-CGTGACGCCGGTGAAGAAT-30; reverse: 50-CGTGCACTCGGCCGTCTT-30), $4.5 \mathrm{nmol}$ dNTPs, $1 \mathrm{U}$ Taq polymerase, $3 \mu \mathrm{L}$ buffer $10 x$ and $1 \mu \mathrm{L}$ of DNA extracted from individual sand flies. Thirty cycles were necessary for amplification (denaturation $30 \mathrm{~s}$ at $94^{\circ} \mathrm{C}$, annealing $1 \mathrm{~min}$ at $62^{\circ} \mathrm{C}$ and elongation $1 \mathrm{~min}$ at $72^{\circ} \mathrm{C}$ ), followed by $10 \mathrm{~min}$ at $72^{\circ} \mathrm{C}$. All of the amplification reactions were analysed by $1 \%-1.5 \%$ agarose gel electrophoresis, followed by ethidium bromide staining and visualization under UV light. Standard DNA fragments (100 bp ladder, Fermentas) were used to permit sizing.

\section{Restriction of cpb PCR fragments}

This assay was developed by Oshaghi et al. [22] for discrimination of $L$. infantum from $L$. donovani using restriction of $\mathrm{cpb}$ PCR products by DraIII enzyme. Digestion was carried out in a total volume of $20 \mu \mathrm{L}$, with approximately $10 \pm 5 \mathrm{ng}$ of DNA (10-15 $\mu \mathrm{L}$ PCR products) and $5 \mathrm{U}$ of restriction enzyme in the recommended buffer, overnight at the recommended temperature. Restriction fragments were separated at $120 \mathrm{~V}$ for $1 \mathrm{~h}$ in $2 \%$ agarose gel and ethidium bromide staining.

\section{Results}

Altogether, 2088 sand flies were collected from the above-mentioned villages (3 locations per village) and identified. P. sergenti (Parrot) and P. kandelakii (Shchurenkova) were the most prevalent Phlebotomus species found at $31.3 \%$ and $10 \%$ respectively. Other species included P. papatasi (Scopoli) (6.3\%), P. caucasicus (Marzinovsky) (5.3\%), P. major (Annandale) (5.2\%), P. halepensis (Theodor) (0.9\%),P. longidactus (Parrot) (0.8\%),Sergentomyia sumbarica (Perfil'ev) (27.5\%) and S. sintoni (Pringle) (12.7\%). Among the sand flies collected $31 \%$ were female and the rest were male. Abdominal examination of female specimens showed $70 \%$ were unfed, $15 \%$ semigravid, $10 \%$ gravid and $5 \%$ blood fed (Table 1). In total 260 females ( $40 \%$ of all collected females) were selected for PCR examination and parasite detection.

Of 260 female specimens tested for promastigote infection, only 2 out of 59
(3.4\%) P. kandelakii were found naturally infected with $L$. infantum/L. donovani. This was observed in the $\mathrm{kDNA}$ nested-PCR amplification assays where a 680 bp PCR band was produced. This length of PCR in the system is assigned to $L$. infantum/L. donovani. This was then confirmed by ITS1 PCR-RFLP using HaelII enzyme. The diagnostic fragments are 220 and $140 \mathrm{bp}$ for L. major, 200, 80 and $60 \mathrm{bp}$ for $L$. infantum/L. donovani, and 2 fragments of 200 and 60 bp are for L. tropica. The result of PCRRFLP revealed 200, 80 and 60 bp bands, which is indicative of $L$. infantum $/ L$. donovani (Figure 1).

To discriminate between L. infantum and L. donovani, cpb PCR was performed and a $702 \mathrm{bp}$ was produced for both specimens. The restriction digestion of the cpb PCR products with the enzyme DraIII gave intact PCR products (702 bp) that were associated with the presence of $L$. infantum (Figure 2).

Of 59 female P. kandelakii, 2 (3.4\%) were found naturally infected with $L$. infantum. One infected specimen was caught from a living room and the other from a yard in Starkhi village.

This is the first report of $P$. kandelakii naturally infected with $L$.infantum in Shirvan district, northern Khorassan province, north-eastern Islamic Republic of Iran.

\begin{tabular}{lccccccccc}
\hline Table1 Species composition of sand flies in Shirvan district, north-eastern Islamic Republic of Iran, 2010 & \\
Species & Male & Female & Unfed & Fed & $\begin{array}{c}\text { Semi- } \\
\text { gravid }\end{array}$ & Gravid & Total \\
& No. & No. & No. & No. & No. & No. & No. & $\%$ \\
Phlebotomus kandelakii & 150 & 59 & 40 & 3 & 6 & 10 & 209 & 10 \\
P. major & 99 & 10 & 10 & 0 & 0 & 0 & 109 & 5.2 \\
P. halepensis & 19 & 0 & 0 & 0 & 0 & 0 & 19 & 0.9 \\
P. papatasi & 81 & 50 & 34 & 4 & 5 & 7 & 131 & 6.3 \\
P. sergenti & 451 & 203 & 140 & 10 & 30 & 23 & 654 & 31.3 \\
P. longidactus & 17 & 0 & 0 & 0 & 0 & 0 & 17 & 0.8 \\
P. caucasicus & 110 & 0 & 0 & 0 & 0 & 0 & 110 & 5.3 \\
Sergentomyia sintoni & 125 & 140 & 100 & 7 & 21 & 12 & 265 & 12.7 \\
S. sumbarica & 388 & 186 & 130 & 8 & 35 & 13 & 574 & 27.5 \\
Total & $1440(69 \%)$ & $648(31 \%)$ & $454(70 \%)$ & $32(5 \%)$ & $97(15 \%)$ & $65(10 \%)$ & 2088 & 100 \\
\hline
\end{tabular}




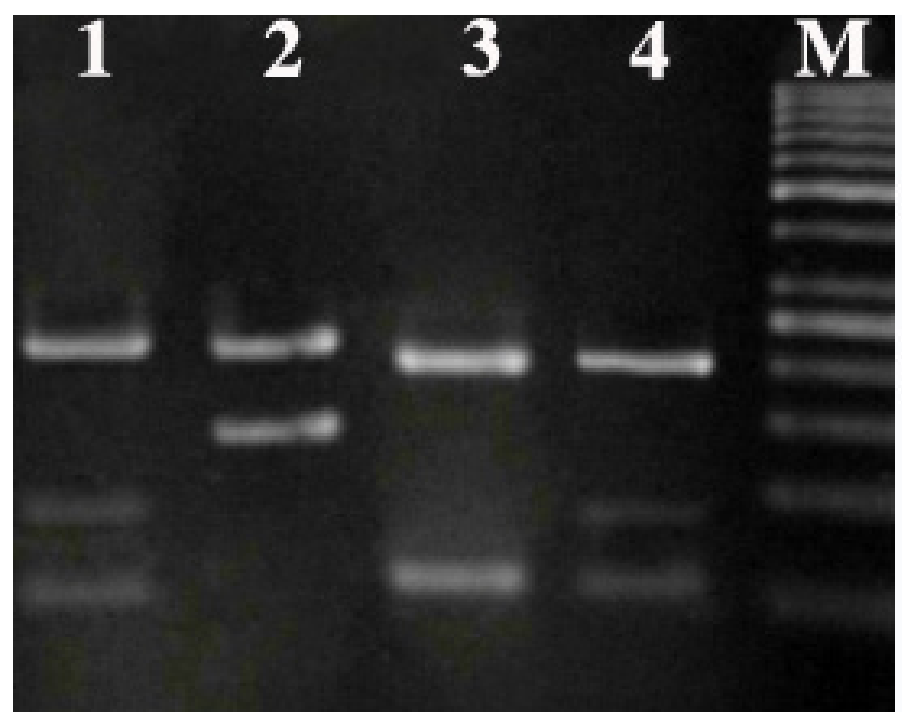

500 bp

Figure 1 ITS1-rDNA PCR-RFLP analysis of selected strains of Leishmania species using Haell restriction enzyme. Lanes: M, 100 bp ladder (Fermentas); 1, infected Phlebotomus kandelakii to L. infantum; 2, L. major (MHOM/IR/75/ER: serial code of the standard $L$. major species at the reference laboratory of the Parasitology Department, Tehran University of Medical Sciences); 3, L. tropica (MHOM/IR/03/ Mash-878); 4, L. infantum (positive control)

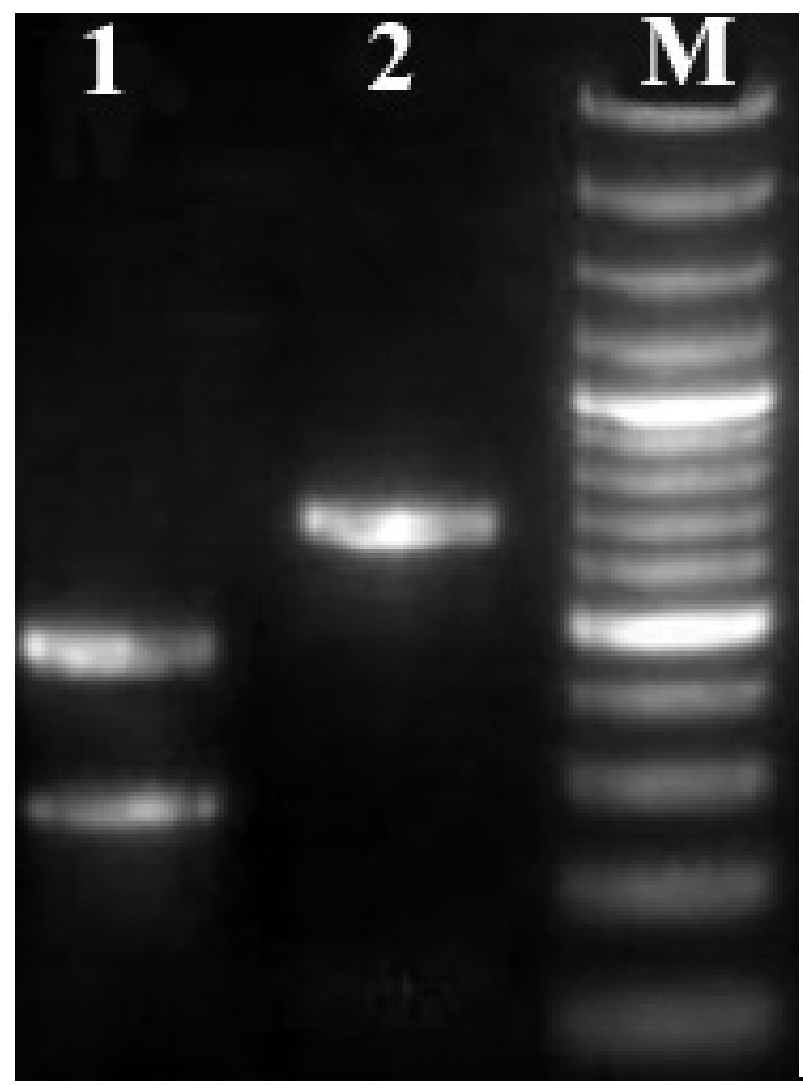

$1000 \mathrm{bp}$

Figure $2 \mathrm{cpbE} / \mathrm{F}$ gene PCR-RFLP analysis of selected strains of Leishmania donovani complex with the use of the restriction enzyme DrallI. Lanes: M, 100 bp ladder (Fermentas); 1, L. donovani: cpbF/cpbE (MHOM/DZ/82/LIPA59); 2, L. infantum from infected Phlebotomus kandelakii

\section{Discussion}

The ecology and epidemiology of leishmaniasis are important measures for management and planning of disease control. Entomological surveys accompanied by epidemiological data provide essential information for the design of control programmes of the disease.

Several epidemiological and entomological findings, including anthropophily, common infection of the sand flies with the same Leishmania parasite found in the patients in the same places, suggest the capacity of the sand fly to be a vector [17]. In the current study, infection of $P$. kandelakii with L. infantum was confirmed using sensitive PCR technique.

Isoenzyme detection methods provide the gold-standard for identifying species and reference strains of Leishmania, but this method has disadvantage as it requires the culture of a large number of parasites and primary isolates can easily become contaminated, or a mixed infection can yield only the strain that grows fast in laboratory conditions [23]. The highly sensitive technique of PCR has been used before to detect Leishmania in New and Old World sand flies $[24,25]$. Some species of sand flies belong to subgenus Larroussius and are potential vectors of VL in the Mediterranean basin. Aransay et al. in 2000 used this method in Greece for detection of Leishmania infection in P. (Lar.) neglectus, P. (Lar.) tobbi, P. (Lar.) simici, and P.(Para.) alexandri using a semi-nested PCR technique [9]. Based on PCR detection and sequencing of the parasite cpb, Oshghi et al. in 2009 confirmed $P$. perfiliewi transcaucasicus was circulating both $L$. donovani and L. infantum in north-western Islamic Republic of Iran [11]. In our study species-specific PCR of the cpbE/F gene against the 2 positive ITS rDNA specimens of $P$. kandelakii revealed the presence of $L$. infantum in the sand fly. This method is able to separate infection of sand flies with L. donovani 
complex, i.e. to distinguish L. donovani from L. infantum [22].

P. kandelakii has been reported as a primary and proven vector of VL in an important focus of disease, i.e. northwestern Islamic Republic of Iran [9]. This species has been reported in central Asia, Afghanistan, Islamic Republic of Iran, Lebanon, Turkey and the former USSR [23]. P. kandelakii is very hydrophilic, moderately thermophilic and bites man and large animals easily in Afghanistan [26,27]; it is also considered to be a vector of VL in the South Caucasus region [27].

The results of blood meal analyses indicate the P. kandelakii collected in north-western Islamic Republic of Iran are strongly anthropophilic with $32.8 \%$ containing human blood and 21.2\% canine blood. P. kandelakii may also therefore play an important role in the transmission of VL to dogs, which are the main domestic reservoir of disease [28]. Among the female sand flies examined, only 2 specimens of $P$. kandelakii were found naturally infected by L. infantum parasites. Both infected sand flies were empty and it seems the females' longevity was enough to complete the parasite's cycle in its body.

Based on its natural infection with L. infantum and the fact that it was the only species found infected with $L$. infantum, we conclude that P. kandelakii can be incriminated as the vector of VL in north-eastern Islamic Republic of Iran. This is the first detection of $L$. infantum in P. kandelakii from Shirvan district, north-eastern Islamic Republic of Iran. It is recommended that the detailed biology of P. kandelakii, particularly its host preference, should be investigated using the available molecular method. This, in combination with other ecological data, could be used in vector control measures of VL in the region [29].

\section{Acknowledgements}

This study received financial support from the School of Public Health, Tehran University of Medical Sciences, Project No.6602.

We are grateful to Dr N. Nikparast, the Health Deputy of North Khorassan University of Medical Sciences, for providing facilities to conduct this research. Furthermore, our thanks go to Mr M. Heydarpour and Mr S. Azari for their cooperation in the field sampling.

\section{References}

1. Burden of disease in DALYS by cause, sex and mortality stratum in WHO regions estimates for 2001. Geneva, World Health Organization, 2002:92-197.

2. Shyma S, Rai M. Laboratory diagnosis of visceral leishmaniasis. Clinical and Diagnostic Laboratory Immunology, 2002, 9:951-958.

3. Control of the leishmaniases. Report of a WHO Expert Committee. Geneva, World Health Organization, 1990 (Technical Report Series, No. 793).

4. Lachaud L et al. Comparison of six PCR methods using peripheral blood for detection of canine visceral leishmaniasis. Journal of Clinical Microbiology, 2002, 40:210-215.

5. Rassi Y et al. [Fauna of sandflies in north-western parts of Iran]. Hakim Research Journal, 1999, 3:11-17 [In Farsi].

6. Rassi Y, Firouzi R, Javadian E. Position of visceral leishmaniosis vectors in the Kaleibar focus, East Azarbaijan Province. Modarres. Journal of Medical Science, 2000, 3:9-14.

7. Mohebali $\mathrm{M}$ et al. Seroepidemiological study of visceral leishmaniasis among humans and animal reservoirs in Bushehr province, Islamic Republic of Iran. Eastern Mediterranean Health Journal, 2001, 7:912-917.

8. Nadim A et al. Epidemiological aspects of Kala-azar in MeshkinShahr, IR Iran: Investigation in vectors. Iranian Journal of Public Health, 1992, 21:61-72

9. Rassi Y et al. Phlebotomus (Larroussius) kandelakii, the principle and proven vector of visceral leishmaniasis in north west of Iran. Pakistan Journal of Biological Sciences, 2005, 8:1802-1806.

10. Rassi Y et al. Phlebotomus perfiliewi transcaucasicus, a Vector of Leishmania infantum in Northwestern Iran. Journal of Medical Entomology, 2009, 46:1094-1098.

11. Oshaghi MA et al. Phlebotomus perfiliewi transcaucasicus is circulating both Leishmania donovani and L. infantum in northwest Iran. Experimental Parasitology, 2009, 123:218-225.
12. Azizi K et al. Phlebotomus (Paraphlebotomus) alexandri: a probable vector of Leishmania infantum in Iran. Annals of Tropical Medicine and Parasitology, 2006, 100:63-68.

13. Azizi $\mathrm{K}$ et al. First detection of Leishmania infantum in Phlebotomus (Larroussius) major (Diptera: Psychodidae) from Iran. Journal of Medical Entomology, 2008, 45:726-731.

14. Sahabi $Z$ et al. A preliminary report on the natural leptomonad infection of Phlebotomus major in an endemic focus of visceral leishmaniasis (VL) in Fars province, Southern of IR Iran. Iranian Journal of Public Health, 1992, 21:87-94.

15. Seyyedi-Rashti MA, Sahabi Z, Kanani Notash A. Phlebotomus (Larroussius) keshishiani Shchurenkova, another vector of visceral leishmaniasis in Iran. Iranian Journal of Public Health, 1995, 24:23-30.

16. Rassi $\mathrm{Y}$ et al. First report on natural promastigote infection of Phlebotomus caucasicus in a new focus visceral leishmaniasis in north west of Iran. Iranian Journal of Public Health, 2004, 33:70-72.

17. Theodor O, Mesghali A. On the phlebotomine of Iran. Journal of Medical Entomology, 1964, 1:285-300.

18. Noyes HA et al. A nested-PCR-based schizodeme method for identifying Leishmania kinetoplast minicircle classes directly from clinical samples and its application to the study of the epidemiology of Leishmania tropica in Pakistan. Journal of Clinical Microbiology, 1998, 36:2877-2881.

19. El Tai NO et al. Genetic heterogeneity of ribosomal internal transcribed spacer in clinical samples of Leishmania donovani spotted on filter paper as revealed by single-strand conformation polymorphisms and sequencing. Transactions of the Royal Society of Tropical Medicine and Hygiene, 2000, 94:575-579.

20. Oshaghi MA et al. Vector incrimination of sand flies in the most important visceral leishmaniasis focus in Iran. American Journal of Tropical Medicine and Hygiene, 2009, 81:572-577. 
21. Hide M, Bañuls AL. Species-specific PCR assay for L. Infantum/L. donovani discrimination. Acta Tropica, 2006, 100:241-245.

22. Oshaghi MA et al. Development of species-specific PCR and PCR-restriction fragment length polymorphism assays for $L$. infantum/L. donovani discrimination. Experimental Parasitology, 2009, 122:61-65.

23. Alvar J, Barker JR. Molecular tools for epidemiological studies and diagnosis of leishmaniasis and selected other parasitic disease. Transactions of the Royal Society of Tropical Medicine and Hygiene, 2002, 96:S1-S250.

24. Mukherjee $\mathrm{S}$ et al. Short report: Leishmania DNA in Phlebotomus and Sergentomyia species during a kala-azar epidemic. American Journal of Tropical Medicine and Hygiene, 1997, 57:423-425.

25. De Bruijn MH, Barker DC. Diagnosis of new world leishmaniasis: specific detection of species of the Leishmania brasiliensis complex by amplification of kinetoplast DNA. Acta Tropica, 1992, 52:45-58.

26. Killick-Kendrick R. Phlebotomine vectors of the leishmaniases: a review. Journal of Medical and Veterinary Entomology, 1990, 4:1-24.

27. Lewis DG. The phlebotomine sandflies (Diptera: Psychodidae) of the Oriental region. London, British Musuem (Natural History), 1978.:57.

28. Maleki-Ravasan $\mathrm{N}$ et al. Blood meal identification in fieldcaptured sand flies: Comparison of PCR-RFLP and ELISA assays. Iranian Journal of Arthropod-Borne Diseases, 2009, 3:8-18.

29. Ramezani Awal Riabi $\mathrm{H}$ et al. [Efficacy of deltamethrin-impregnated dog collars against sandflies at endemic focus of Bojnord district, north Khorassan province]. Ofogh-E-Danesh, 2009, 15:20-29 [in Farsi].

\section{Handbook for integrated vector management}

Integrated vector management (IVM) is a rational decision-making process for optimal use of resources for vector control. The aim of the IVM approach is to contribute to achievement of the global targets set for vector-borne disease control, by making vector control more efficient, cost-effective, ecologically sound and sustainable. The handbook for integrated vector management presents an operational framework to guide managers and those implementing vectorborne disease control programmes in designing more efficient, cost-effective systems. The handbook discusses the policy and institutional framework for IVM; planning and implementation of IVM; capacity-building, including human resource development; the core functions and essential competence required for IVM at central and local levels; the elements and processes of IVM; and a comprehensive framework for monitoring and evaluation of IVM, including indicators and methods for measuring process, outcomes and impact.

Further information about this and other WHO publications is available at: http://www.who.int/publications/en/ 